Chinese Journal of Organic Chemistry

NOTE

\title{
6-取代-3-胺基雌激素的高效合成
}

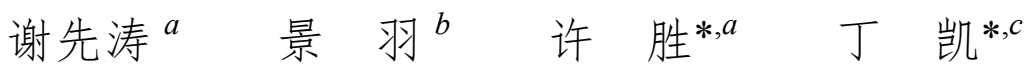

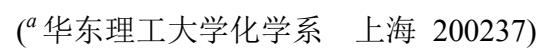 \\ $\left({ }^{b}\right.$ 上海师范大学资源化学实验室 上海 200234) \\ ( ${ }^{c}$ 中国科学院上海有机化学研究所 天然产物有机合成化学重点实验室 上海 200032)
}

\begin{abstract}
摘要 3-胺基雌激素是潜在的雌激素药物, 传统的合成方法以雌酚酮为原料, 难以进行多样性导向的快速合成. 以 19羟基雄甾-4-烯-3,7-二酮为原料, 利用串联的 retro-aldol 芳构化/分子内亲核加成高效合成了 6-取代-3-胺基雌激素. 该方 法试剂易得，操作简单，可一步反应同时导入两个取代基.
\end{abstract}

关键词＼cjkstart雌激素; 烯胺化; 芳构化; 分子内亲核加成

\section{Efficient Synthesis of 6-Substitued-3-aminoestrogens}

\author{
Xie, Xiantao ${ }^{a} \quad$ Jin, $\mathrm{Yu}^{b} \quad \mathrm{Xu}$, Sheng ${ }^{*, a} \quad \mathrm{Ding} \mathrm{Kai}^{*, c}$ \\ ( ${ }^{a}$ Department of Chemistry, East China University of Science and Technology, Shanghai 200237) \\ ( ${ }^{b}$ Laboratory of Resource Chemistry, Shanghai Normal University, Shanghai 200234) \\ ( ${ }^{c}$ CAS Key Laboratory of Synthetic Chemistry of Natural Substances, Shanghai Institute of Organic Chemistry, \\ Chinese Academy of Sciences, Shanghai 200032)
}

\begin{abstract}
Aminoestrogens are promising estrogen drugs. Traditional synthesis of the estrogens used estrone as a raw material, however, was not suitable for diversity-oriented synthesis. Herein, an efficient synthesis of 6-substitued-3-aminoestrogens from easily available 19-hydroxy-androst-4-ene-3,17-dione via a tandem retro-aldol aromatization/intermolecular nucleophilic addition is presented. The method featured easily available reagents, simple operation and introduction of two substituents in one step.
\end{abstract}

Keywords estrogen; enamination; aromatization; intramolecular nucleophilic addition

甾体雌激素类药物具有广泛的生理活性和药用价 值，长期吸引了药物化学家以其为基础进行药物设计. 大多数雌激素药物 $\mathrm{A}$ 环为芳香化的六元碳环, 3 位上有 一个酚羟基. 3-胺基雌激素是它们的生物电子等排体, 作为潜在的甾体此激素药物已被用于治疗前列腺癌、乳 腺癌的研究 ${ }^{[1]}$. 从易得的起始原料高效、快速、结构多 样性地合成这类化合物是深入进行药物化学研究的前 提. 目前 3-胺基雌激素合成一般都使用雌酚酮作为原料 (Scheme 1) $)^{[2]}$. 如通过分子内的 Smiles 反应 ${ }^{[2 \mathrm{a} \sim 2 \mathrm{~d}]}$ 或使用 钯催化 $\mathrm{C}-\mathrm{N}$ 偶联反应 ${ }^{[2 \mathrm{e}-2 \mathrm{~g}]}$ 导入 3 位的胺基. 但这些方 法存在产率低、路线长、底物适用面较窄的问题, 难以 快速构建化合物库.

使用具有 4-烯-3-酮结构的甾体化合物为原料, 通
过烯胺化后再芳构化的策略也可合成 3-胺基雌激素 (Scheme 2), 20 世纪 50 60 年代就有相关例子报道 ${ }^{[3]}$. 该策略中使用的起始物比雌酚酮容易衍生化, 非常适合 多样性合成, 但由于原料未商品化, 合成困难, 限制了 这一策略的应用。

19-羟基-4-烯-3,17-二酮雄甾是一种商品化的甾体 中间体, 我们发展了以此化合物为原料, 通过 retroaldol 后期芳构化策略合成甾体雌激素的一种方法 ${ }^{[4]}$. 由 于 A 环的 4-烯 3-酮结构非常容易衍生化, 可快速合成结 构多样性的甾体雌激素. 最近, 我们使用原甲酸酯作为 烯醇化试剂, 一步合成了 3 位醚化的雌激素 ${ }^{[4 \mathrm{~b}]}$. 设想如 果在芳构化前发生烯胺化, 有可能一步获得 3 位胺化的 雌激素(Scheme 3).

\footnotetext{
* Corresponding authors. E-mail: xusheng@ecust.edu.cn; dingkai@mail.sioc.ac.cn

Received October 4, 2016; revised November 8, 2016; published online November 22, 2016.

Project supported by the National Natural Science Foundation of China (Nos. 20902098, U1362111).

国家自然科学基金(Nos. 20902098, U1362111)资助项目.
} 


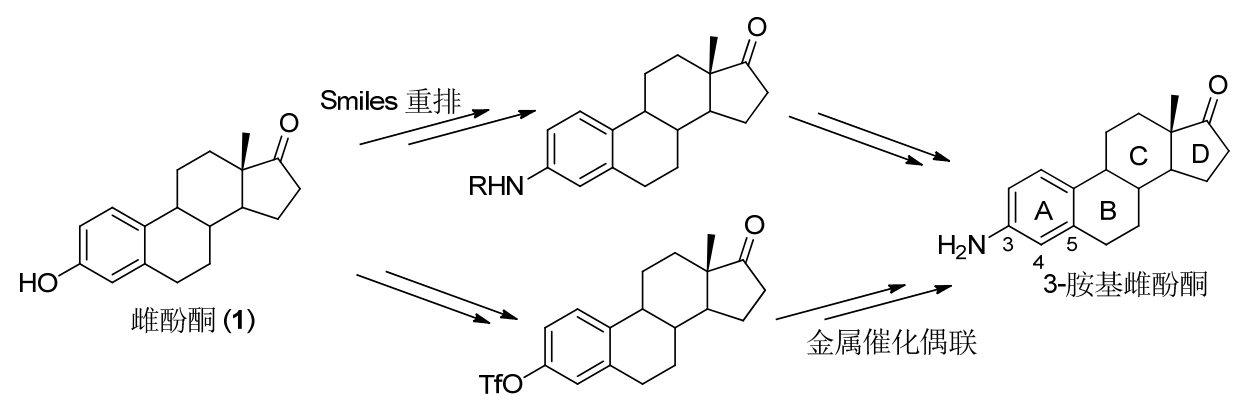

图式 1 从雌酚酮合成 3-胺基雌激素

Scheme 1 Synthesis of 3-aminoestrogens from estrone

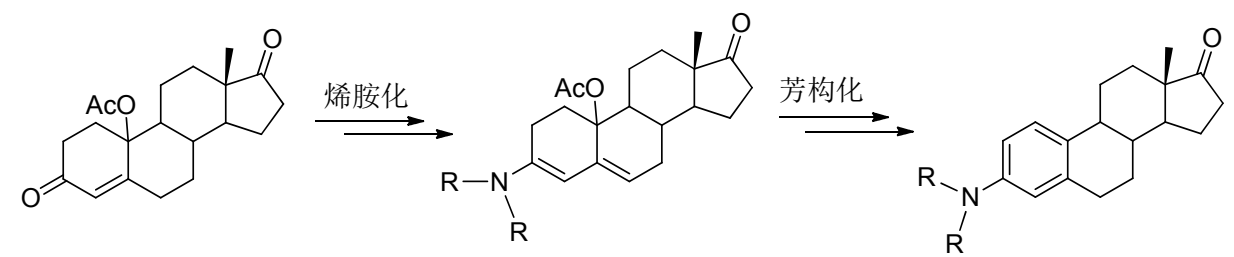

图式 2 从 4-烯-3-酮甾体合成 3-胺基雌激素

Scheme 2 Synthesis of 3-aminoestrogens from 4-en-3-one steroids

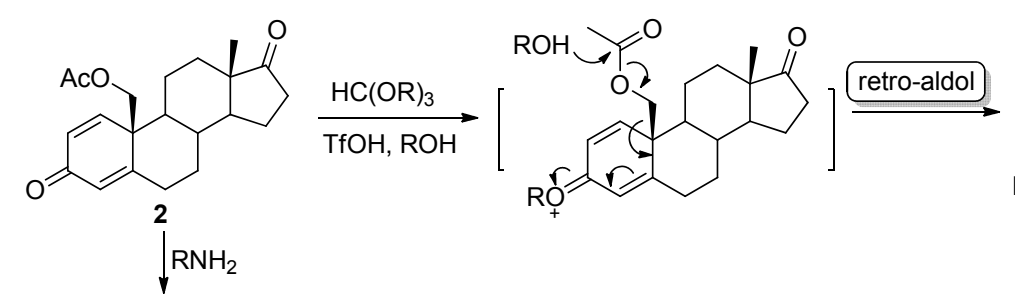<smiles>[R6]Oc1ccc2c(c1)CCC1C2CCC2(C)C(=O)CCC12</smiles>

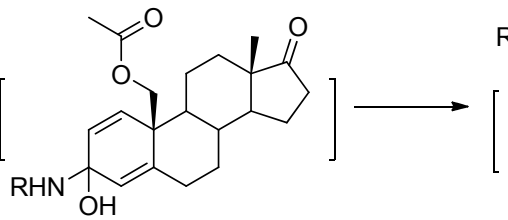<smiles>CC(=O)OCC1(CCN)CCC2C(CCC3(C)C(=O)CCC23)C1CCCN</smiles><smiles>[R]Nc1ccc2c(c1)CCC1C2CC[C@@]2(C)C(=O)CCC12</smiles>

图式 3 由 19-差基甾体化合物合成 3-胺基雌激素

Scheme 3 Synthesis of 3-aminoestrogens from 19-hydroxy steroid

\section{1 结果与讨论}

我们按先前报道的方法合成了底物二烯酮 $\mathbf{2}^{[4]}$. 在 一些常规的烯胺化条件下 ${ }^{[3]}$ 测试了伯胺与二烯酮 2 的反 应, 但是遗憾的是产物为雌酚酮 1 . 显然, 小位阻的伯 胺进攻 19 位酯基而不是 3 位羰基, 导致在烯胺化前直接 发生了 retro-aldol 芳构化反应. 改用位阻较大的仲胺, 当用甲醇作溶剂时, 获得了少量胺化物 $\mathbf{3}$, 但主产物仍 是雌酚酮 1 (表 1, Entry 1). 使用更为稳定的酯基保护 19 位羟基时, 反应明显变慢, 胺化物 $\mathbf{3}$ 的产率没有明显提 高, 仍有大量雌酚酮形成(Entry 2). 我们猜想甲醇在碱 性条件下形成小位阻的亲核试剂, 导致在烯胺化前发生 芳构化, 改用无亲核能力的惰性溶剂有可能避免这一问 题. 苯和二氧六环作溶剂时, 反应速度明显变慢, 但对 反应选择性没有太大影响(Entries 3,4). 意外的是, 使用
二甲基亚砜(DMSO)为溶剂时，主要形成一个未知产物 (Entry 5), 核磁分析显示是一对异构体的混合物, 分子 的 A 环发生了芳构化, 且具有吡咯烷的结构, 但是仍然 存在一个甲基酮结构. 尽管这对异构体无法通过柱层析 分离, 通过重结晶幸运地获得了其中一个异构体的纯品 4a, 通过单晶衍射(图 1), 确认为 6 位连接一个乙酰基的 3-胺基产物. 此异构体在室温中性条件下稳定, 可长期 保存而不发生差向异构化. 进一步的研究发现，使用大 位阻的醇为溶剂，也可以得到这一产物，而且反应速度 更快(Entries 6,7). 经过反应优化，以 70\%的产率获得 6乙酰基-3-胺基雌酮 (4, Entry 8). 也尝试使用其他一些仲 胺, 如二乙胺、甲基苯胺、二烯丙基胺等，但在此条件 下仅回收原料，说明胺的位阻对反应至关重要，太小会 直接生成䧳酚酮, 太大又无法发生烯胺化反应. 
表 1 反应条件优化 ${ }^{a}$

Table 1 Optimization of reaction conditions

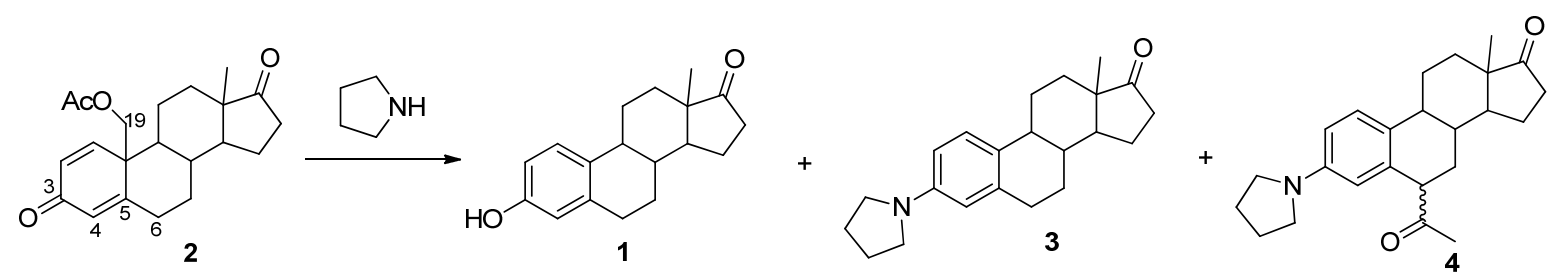

\begin{tabular}{|c|c|c|c|c|c|c|c|c|}
\hline \multirow{2}{*}{ Entry } & \multirow{2}{*}{ 吡咯烷/equiv. } & \multirow{2}{*}{ 溶剂 } & \multirow{2}{*}{ 温度/ ${ }^{\circ} \mathrm{C}$} & \multirow{2}{*}{ 时间 $/ \mathrm{h}$} & \multicolumn{3}{|c|}{ 产率/\% } & \multirow{2}{*}{ 转化率 $b / \%$} \\
\hline & & & & & 1 & 3 & 4 & \\
\hline 1 & 2 & $\mathrm{MeOH}$ & 60 & 4 & 60 & 26 & - & 100 \\
\hline 2 & $2^{c}$ & $\mathrm{MeOH}$ & 60 & 20 & 24 & 31 & & 98 \\
\hline 3 & 2 & Benzene & 80 & 16 & 21 & 25 & - & 50 \\
\hline 4 & 2 & Dioxane & 100 & 12 & - & 8 & 7 & 40 \\
\hline 5 & 2 & DMSO & 100 & 12 & - & 8 & 44 & 55 \\
\hline 6 & 2 & $\mathrm{EtOH}$ & 80 & 7 & 8 & 14 & 29 & 95 \\
\hline 7 & 16 & $i$-PrOH & 80 & 16 & - & 10 & 63 & 100 \\
\hline 8 & 4 & $i$-PrOH & 100 & 25 & - & - & 70 & 100 \\
\hline
\end{tabular}

$\bar{a}$ 原料(0.5 mmol), 溶剂 $(5 \mathrm{~mL}) ;{ }^{b}$ 分离收率; ${ }^{c} 19$ 位羟基保护基为三甲基乙酰基(Piv).

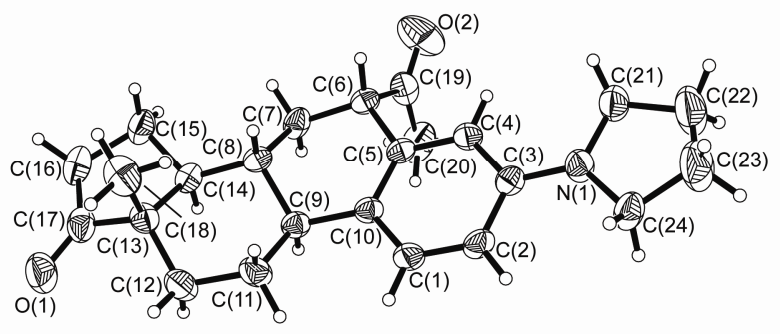

图 1 化合物 $4 \mathrm{a}$ 的晶体结构(6- $\alpha$-异构体)

Figure 1 Crystal structure of compound $4 a$ (6- $\alpha$-isomer)

以上结果说明，除了前面假设的协同的 retro-aldol 芳构化机理 ${ }^{[4]}$, 在有竞争性反应存在下, 芳构化也可分 步进行. 可能的机理如下, 在惰性溶剂中, 3 位羰基与胺 反应形成烯胺 ${ }^{[5 a]}$, 然后发生分子内的亲核加成, 导致 19 位酯基保护基转移到 6 位 ${ }^{[5 b]}$, 同时发生芳构化反应. 这 个分子内的加成反应是立体专一性的, 但是由于芳环和 羰基共同的活化作用, 6 位极易发生异构化, 在过量碱 的存在下，最终得到异构体的混合物(Scheme 4).

在甾体的 6 位导入取代基, 可显著改变甾体药物的 活性. 常见的 6 位取代的甾体药物有抗癌药 exemestane (依西美坦)和避孕药 megestrol(甲地孕酮). 合成可通过 Vilsmeier 反应或 Mannich 反应在 C-6 位引入次甲基, 再 进一步转化获得 ${ }^{[6]}$. 但这些方法一般多用于甲基的导入, 通用性不强. 上述方法如果具有普适性, 对反应底物 19 位保护基进行简单的替换就可以导入各种结构的 6 位取 代基，对这些取代基进行简单的衍生化就可快速建立一 个3-胺基-6 取代的雌激素库. 为此我们合成了一系列 19 位不同酯基保护的底物，考察了反应的普适性(表 2).

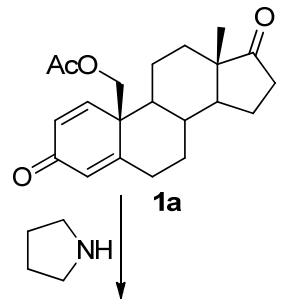

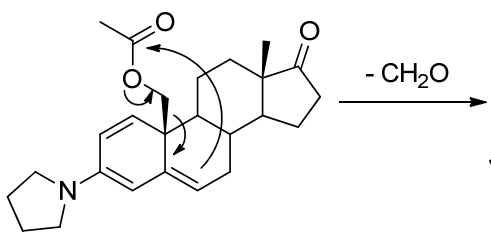

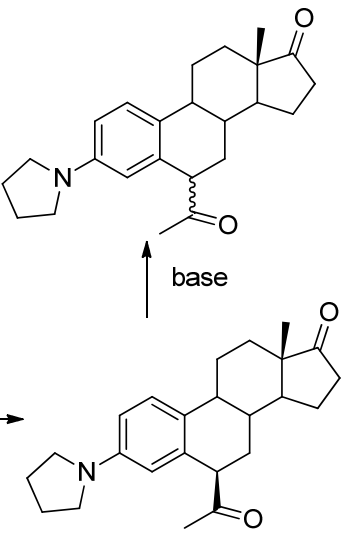

图式 4 合成 3-胺基 6-酰基雌激素的机理

Scheme 4 Proposed mechanism of synthesis of 3-amino-6-acylestrogens

甲酰基非常容易脱除, 主要得到䧳酚酮(Entry 1). 其它 的脂肪酯基获得的结果与乙酰基类似(Entries $3 \sim 8$ ), 形 成了对应的 C-6 取代产物，且产率接近. 当取代基为特 戊酰基时(Entry 9), 反应 $22 \mathrm{~h}$ 仍未转化完全, 且无 6-取 代产物形成, 可能由于位阻过大，难以发生分子内亲核 取代所致. 当保护基是烯基酯时(Entries 10,11), 原料完 全消失, 但是没有分离得到目标产物, 只获得少量无法 鉴别的混合物, 可能是由于丙烯酸酯引发的副反应所 致. 保护基是碳酸酯时(Entry 12), 反应也可顺利发生, 得到 6 位增加一个碳原子的产物. 使用芳基酯保护基时 (Entries 13,14), 同样获得了目标产物，但是由于芳基对 羰基的钝化作用, 反应进行较慢, 且产率较低. 
表 2 反应底物拓展 ${ }^{a}$

Table 2 Scope of the substrates
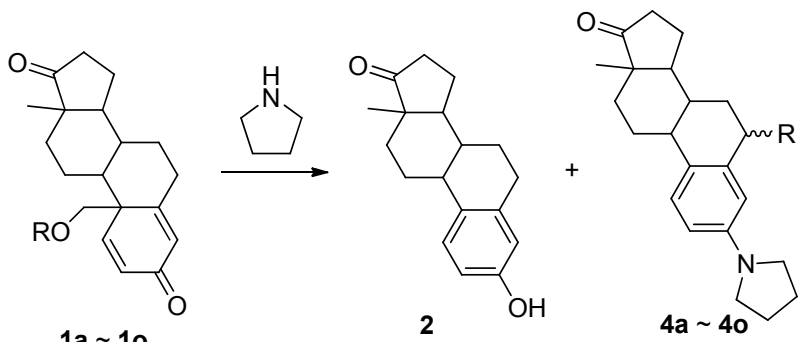

$1 \mathrm{a} \sim 10$

\begin{tabular}{cclcc}
\hline Entry & Compd. & \multicolumn{1}{c}{$\mathrm{R}$} & \multicolumn{1}{c}{$t / \mathrm{h}$} & $\mathrm{Yield}^{b} / \%$ (Conv./\%) \\
\hline 1 & $\mathbf{1 a}$ & $\mathrm{Ac}$ & 8 & 70 \\
2 & $\mathbf{1 b}$ & $\mathrm{CHO}$ & 20 & - \\
3 & $\mathbf{1 c}$ & $\mathrm{C}_{2} \mathrm{H}_{5} \mathrm{CO}$ & 22 & 52 \\
4 & $\mathbf{1 d}$ & $\mathrm{CH}_{3}\left(\mathrm{CH}_{2}\right)_{2} \mathrm{CO}$ & 22 & 50 \\
5 & $\mathbf{1 e}$ & $\left(\mathrm{CH}_{3}\right)_{2} \mathrm{CHCO}$ & 22 & 51 \\
6 & $\mathbf{1 f}$ & $\mathrm{CH}_{3}\left(\mathrm{CH}_{2}\right)_{3} \mathrm{CO}$ & 22 & 59 \\
7 & $\mathbf{1 g}$ & $\left(\mathrm{CH}_{3}\right)_{2} \mathrm{CHCH}_{2} \mathrm{CO}$ & 22 & 52 \\
8 & $\mathbf{1 h}$ & $\mathrm{CH}_{3}\left(\mathrm{CH}_{2}\right)_{8} \mathrm{CO}$ & 22 & 59 \\
9 & $\mathbf{1 i}$ & $\mathrm{Piv}_{2}$ & 22 & $0(41)$ \\
10 & $\mathbf{1 j}$ & $\mathrm{CH}_{2}=\mathrm{CHCO}_{1}$ & 22 & - \\
11 & $\mathbf{1 k}$ & $\mathrm{PhCH}=\mathrm{CHCO}$ & 22 & - \\
12 & $\mathbf{1 1}$ & $\mathrm{MeOCO}$ & 7 & 48 \\
13 & $\mathbf{1 m}$ & $\mathrm{Bz}$ & 32 & 40 \\
14 & $\mathbf{1 n}$ & $1-\mathrm{Naphthoyl}$ & 22 & $25(87)$ \\
\hline
\end{tabular}

${ }^{a}$ Substrate $(0.5 \mathrm{mmol})$, pyrrolidine $(2 \mathrm{mmol}), i-\operatorname{PrOH}(5 \mathrm{~mL}), 100{ }^{\circ} \mathrm{C} ;{ }^{b}$ isolated yield.

\section{2 结论}

提供了一种以 19 羟基甾体化合物为原料, 通过后 期芳构化策略高效合成 6-酰基-3-胺基雌激素的方法. 该方法操作简单, 原料易得, 一步可在 3 位引入胺基和 6 位引入酰基. 值得一提的是, 简单改变 19 位的保护基 团就可以快速地合成多种 6 位取代的产物, 为这类具有 生理活性的甾体化合物的多样化合成提供了有效的途 径. 以此工作为基础, 后继的衍生化和生物活性测试正 在进行中.

\section{3 实验部分}

\section{1 仪器与试剂}

${ }^{1} \mathrm{H}$ NMR 和 ${ }^{13} \mathrm{C}$ NMR 由 Bruker-AM 400 型或 Bruker-AM 300 型核磁共振仪测定, 若无特别注明, 均 以 $\mathrm{CDCl}_{3}$ 作溶剂, TMS 为内标; ESI-LRMS 采用美国 Agilent 1100LCMSD SL 液相色谱-质谱联用仪和 Agilent 1200/G6100A 液质联用仪测定; ESI-HRMS 使用 Bruker Daltonics FTMS-7 质谱仪进行测定.

原料 19-羟基雄甾-4-烯-3,17-二酮由浙江仙琚制药 有限公司提供提供. 1,4-二烯-3 酮化合物 $1 \mathrm{a} \sim 10$ 按文献 [4]报道的方法合成. 试剂均经纯化. 薄层色谱(TLC)采
用 GF254 高效板，显色剂为紫外灯照射、碘缸显色或 $10 \%$ 磷钼酸乙醇溶液; 柱层析用硅胶 H (200 300 目)作 为固定相.

\section{2 取代-3-胺基雌酚酮 3, 4a 40 的合成}

$0.5 \mathrm{mmol}$ 化合物溶于 $5 \mathrm{~mL}$ 无水溶剂中，氩气保护 下, 加入无水吡咯烷 $(1.7 \mathrm{~mL}, 2 \mathrm{mmol}), 100{ }^{\circ} \mathrm{C}$ 摚拌, 薄 层色谱(TLC)监测反应, 反应结束后减压除去溶剂, 剩 余物直接柱层析分离得到产品.

3-四氢吡咯基-1,3,5(10)-三烯-17-酮-雌甾(3): m.p. $162 \sim 163{ }^{\circ} \mathrm{C} ;[\alpha]_{\mathrm{D}}^{25}+55.6\left(c 0.5, \mathrm{CHCl}_{3}\right) ;{ }^{1} \mathrm{H}$ NMR $(400$ $\left.\mathrm{MHz}, \mathrm{CDCl}_{3}\right) \delta: 7.16(\mathrm{~d}, J=8.5 \mathrm{~Hz}, 1 \mathrm{H}), 6.43(\mathrm{dd}, \quad J=$ 8.4, $2.2 \mathrm{~Hz}, 1 \mathrm{H}), 6.33$ (s, 1H), 3.26 (t, J=6.4 Hz, 4H), 2.89 (d, $J=5.1 \mathrm{~Hz}, 2 \mathrm{H}), 0.90(\mathrm{~s}, 3 \mathrm{H}) ;{ }^{13} \mathrm{C} \mathrm{NMR}(101 \mathrm{MHz}$, $\mathrm{CDCl}_{3}$ ) $\delta: 221.3,146.3,137.0,126.8,126.0,111.8,109.8$, 77.3, 77.0, 76.7, 50.4, 48.1, 47.7, 43.9, 38.7, 35.9, 31.6, 29.9, 26.8, 26.0, 25.4, 21.6, 13.9; ESI-HRMS calcd for $\mathrm{C}_{22} \mathrm{H}_{30} \mathrm{NO} 324.2322$, found 324.2320 .

6-乙酰基-3-四氢吡咯基-1,3,5(10)-三烯-17-酮-䧳甾 (4a): $3: 7$ 混合物, 浅绿色固体; ${ }^{1} \mathrm{H}$ NMR (400 MHz, $\left.\mathrm{CDCl}_{3}\right) \delta: 7.21(\mathrm{~d}, J=8.6 \mathrm{~Hz}, 1 \mathrm{H}), 6.51(\mathrm{dd}, J=8.5,2.1$ $\mathrm{Hz}, 1 \mathrm{H}), 6.20$ (d, $J=2.2 \mathrm{~Hz}, 0.3 \mathrm{H}), 6.17$ (d, $J=2.0 \mathrm{~Hz}$, $0.7 \mathrm{H}), 3.93(\mathrm{t}, J=8.5 \mathrm{~Hz}, 0.7 \mathrm{H}), 3.86(\mathrm{~d}, J=5.6 \mathrm{~Hz}$, $0.3 \mathrm{H}), 3.24 \sim 3.21(\mathrm{~m}, 4 \mathrm{H}), 0.90(\mathrm{~s}, 0.9 \mathrm{H}), 0.88(\mathrm{~s}, 2.1 \mathrm{H})$; ESI-HRMS calcd for $\mathrm{C}_{24} \mathrm{H}_{32} \mathrm{NO}_{2}$ 366.2428, found 366.2420 .

4a: 6- $\alpha$-异构体，无色晶体. m.p. $157 \sim 158{ }^{\circ} \mathrm{C}$; $[\alpha]_{\mathrm{D}}^{25}+222.5\left(c\right.$ 1.00, $\left.\mathrm{CHCl}_{3}\right) ;{ }^{1} \mathrm{H} \mathrm{NMR}(400 \mathrm{MHz}$, $\left.\mathrm{CDCl}_{3}\right) \delta: 7.21(\mathrm{~d}, J=8.6 \mathrm{~Hz}, 1 \mathrm{H}), 6.50(\mathrm{dd}, J=8.6,2.5$ $\mathrm{Hz}, 1 \mathrm{H}), 6.16$ (d, $J=2.3 \mathrm{~Hz}, 1 \mathrm{H}), 3.92$ (t, $J=8.6 \mathrm{~Hz}, 1 \mathrm{H})$, $3.23(\mathrm{t}, J=6.5 \mathrm{~Hz}, 4 \mathrm{H}), 2.03(\mathrm{~s}, 3 \mathrm{H}), 0.88(\mathrm{~s}, 3 \mathrm{H}) ;{ }^{13} \mathrm{C}$ NMR (101 MHz, $\left.\mathrm{CDCl}_{3}\right) \delta: 220.6,211.0,146.5,133.4$, $127.0,126.4,111.2,111.1,77.4,77.0,76.7,55.5,50.3$, $48.0,47.7,43.0,37.4,35.8,31.3,29.7,26.0,25.8,25.4$, $21.6,13.7$.

6-丙酰基-3-四氢吡咯基-1,3,5(10)-三烯-17-酮-䧳甾 (4c): $3: 7$ 混合物, 浅绿色固体; ${ }^{1} \mathrm{H}$ NMR (400 MHz, $\left.\mathrm{CDCl}_{3}\right) \delta: 7.20(\mathrm{~d}, J=8.6 \mathrm{~Hz}, 1 \mathrm{H}), 6.49$ (dd, $J=8.6,2.2$ $\mathrm{Hz}, 1 \mathrm{H}), 6.19$ (d, $J=2.4 \mathrm{~Hz}, 0.3 \mathrm{H}), 6.14(\mathrm{~d}, J=2.3 \mathrm{~Hz}$, $0.7 \mathrm{H}), 3.96(\mathrm{t}, J=8.5 \mathrm{~Hz}, 0.7 \mathrm{H}), 3.87(\mathrm{~d}, J=6.6 \mathrm{~Hz}$, $0.3 \mathrm{H}), 3.31 \sim 3.14(\mathrm{~m}, 4 \mathrm{H}), 1.07(\mathrm{t}, J=7.2 \mathrm{~Hz}, 0.9 \mathrm{H}), 1.01$ $(\mathrm{t}, J=7.3 \mathrm{~Hz}, 2.1 \mathrm{H}), 0.90(\mathrm{~s}, 0.9 \mathrm{H}), 0.88(\mathrm{~s}, 2.1 \mathrm{H})$; ESI-HRMS calcd for $\mathrm{C}_{25} \mathrm{H}_{34} \mathrm{NO}_{2}$ 380.2584, found 380.2582 .

6-丁酰基-3-四氢吡咯基-1,3,5(10)-三烯-17-酮-䧳甾 (4d): $3: 7$ 混合物, 浅绿色固体; ${ }^{1} \mathrm{H}$ NMR (400 MHz, 
$\left.\mathrm{CDCl}_{3}\right) \delta: 7.20(\mathrm{~d}, J=8.6 \mathrm{~Hz}, 1 \mathrm{H}), 6.50(\mathrm{dd}, J=8.5,2.0$ $\mathrm{Hz}, 1 \mathrm{H}), 6.17(\mathrm{~d}, J=2.2 \mathrm{~Hz}, 0.3 \mathrm{H}), 6.15(\mathrm{~d}, J=2.1 \mathrm{~Hz}$, $0.7 \mathrm{H}), 3.95$ (t, $J=8.3 \mathrm{~Hz}, 0.7 \mathrm{H}), 3.87(\mathrm{~d}, J=6.4 \mathrm{~Hz}$, $0.3 \mathrm{H}), \quad 3.30 \sim 3.15(\mathrm{~m}, 4 \mathrm{H}), \quad 0.93 \sim 0.83(\mathrm{~m}, 6 \mathrm{H})$; ESI-HRMS calcd for $\mathrm{C}_{26} \mathrm{H}_{36} \mathrm{NO}_{2}$ 394.2741, found 394.2737.

6-异丁酰基-3-四氢吡咯基-1,3,5(10)-三烯-17-酮-雌 甾(4e): $3: 7$ 混合物, 浅绿色固体; ${ }^{1} \mathrm{H}$ NMR $(400 \mathrm{MHz}$, $\left.\mathrm{CDCl}_{3}\right) \delta: 7.19(\mathrm{~d}, J=8.5 \mathrm{~Hz}, 1 \mathrm{H}), 6.48(\mathrm{dd}, J=8.5,2.1$ $\mathrm{Hz}, 1 \mathrm{H}), 6.15(\mathrm{~d}, J=2.1 \mathrm{~Hz}, 0.7 \mathrm{H}), 6.13(\mathrm{~d}, J=2.2 \mathrm{~Hz}$, $0.3 \mathrm{H}), 4.09 \sim 4.04(\mathrm{~m}, 1 \mathrm{H}), 3.30 \sim 3.13(\mathrm{~m}, 4 \mathrm{H}), 1.97(\mathrm{~s}$, $6 \mathrm{H}), 1.16(\mathrm{~d}, J=6.9 \mathrm{~Hz}, 0.9 \mathrm{H}), 1.15(\mathrm{dd}, J=9.9,6.8 \mathrm{~Hz}$, 2H), 1.07 (dd, $J=12.5,6.8 \mathrm{~Hz}, 4 \mathrm{H}), 1.05(\mathrm{~d}, J=6.7 \mathrm{~Hz}$, 2.1H), $0.90(\mathrm{~s}, 0.9 \mathrm{H}), 0.88(\mathrm{~s}, 2.1 \mathrm{H})$; ESI-HRMS calcd for $\mathrm{C}_{26} \mathrm{H}_{36} \mathrm{NO}_{2}$ 394.2741, found 394.2740.

6-戊酰基-3-四氢吡咯基-1,3,5(10)-三烯-17-酮-雌甾 (4f): $3: 7$ 混合物, 浅绿色固体; ${ }^{1} \mathrm{H}$ NMR $(400 \mathrm{MHz}$, $\left.\mathrm{CDCl}_{3}\right) \delta: 7.20(\mathrm{~d}, J=8.6 \mathrm{~Hz}, 1 \mathrm{H}), 6.49(\mathrm{dd}, J=8.5,2.2$ $\mathrm{Hz}, 1 \mathrm{H}), 6.17$ (d, $J=2.4 \mathrm{~Hz}, 0.3 \mathrm{H}), 6.14(\mathrm{~d}, J=2.3 \mathrm{~Hz}$, $0.7 \mathrm{H}), 3.95(\mathrm{t}, J=8.4 \mathrm{~Hz}, 0.7 \mathrm{H}), 3.87(\mathrm{~d}, J=6.5 \mathrm{~Hz}$, $0.3 \mathrm{H}), \quad 3.31 \sim 3.14(\mathrm{~m}, 4 \mathrm{H}), \quad 0.96 \sim 0.80(\mathrm{~m}, 6 \mathrm{H})$; ESI-HRMS calcd for $\mathrm{C}_{27} \mathrm{H}_{38} \mathrm{NO}_{2}$ 408.2897, found 408.2894.

6-异成酰基-3-四氢吡咯基-1,3,5(10)-三烯-17-酮-雌 甾(4g): $3: 7$ 混合物, 浅绿色固体; ${ }^{1} \mathrm{H}$ NMR $(400 \mathrm{MHz}$, $\left.\mathrm{CDCl}_{3}\right) \delta: 7.11(\mathrm{~d}, J=8.6 \mathrm{~Hz}, 1 \mathrm{H}), 6.41(\mathrm{dd}, J=8.5,2.0$ $\mathrm{Hz}, 1 \mathrm{H}), 6.07(\mathrm{~d}, J=2.4 \mathrm{~Hz}, 1 \mathrm{H}), 3.86(\mathrm{t}, J=8.3 \mathrm{~Hz}$, $0.7 \mathrm{H}), 3.78(\mathrm{~d}, J=6.4 \mathrm{~Hz}, 0.3 \mathrm{H}), 3.33 \sim 3.07(\mathrm{~m}, 4 \mathrm{H})$, $0.88 \sim 0.70(\mathrm{~m}, 9 \mathrm{H})$; ESI-HRMS calcd for $\mathrm{C}_{27} \mathrm{H}_{38} \mathrm{NO}_{2}$ 408.2897, found 408.2893.

6-癸酰基-3-四氢吡咯基-1,3,5(10)-三烯-17-酮-雌甾 (4h): $3: 7$ 混合物, 浅绿色固体; ${ }^{1} \mathrm{H}$ NMR $(400 \mathrm{MHz}$, $\left.\mathrm{CDCl}_{3}\right) \delta: 7.20(\mathrm{~d}, J=8.6 \mathrm{~Hz}, 1 \mathrm{H}), 6.49(\mathrm{dd}, J=8.5,1.9$ $\mathrm{Hz}, 1 \mathrm{H}), 6.17$ (d, $J=2.3 \mathrm{~Hz}, 0.3 \mathrm{H}), 6.14(\mathrm{~d}, J=2.3 \mathrm{~Hz}$, $0.7 \mathrm{H}), 3.95$ (t, $J=8.4 \mathrm{~Hz}, 0.7 \mathrm{H}), 3.87(\mathrm{~d}, J=6.4 \mathrm{~Hz}$, $0.3 \mathrm{H}), 3.31 \sim 3.15(\mathrm{~m}, 4 \mathrm{H}), 1.33 \sim 1.13(\mathrm{~m}, 12 \mathrm{H}), 0.94 \sim$ $0.82(\mathrm{~m}, 6 \mathrm{H})$; ESI-HRMS calcd for $\mathrm{C}_{32} \mathrm{H}_{48} \mathrm{NO}_{2} 478.3880$, found 478.3668 .

6-甲氧酰基-3-四氢吡咯基-1,3,5(10)-三烯-17-酮-雌 甾(4I): $1: 1$ 混合物, 浅绿色固体; ${ }^{1} \mathrm{H}$ NMR $(400 \mathrm{MHz}$, $\left.\mathrm{CDCl}_{3}\right) \delta: 7.18(\mathrm{t}, J=8.0 \mathrm{~Hz}, 1 \mathrm{H}), 6.55 \sim 6.44(\mathrm{~m}, 2 \mathrm{H})$, $6.36(\mathrm{~s}, 0.5 \mathrm{H}), 4.00(\mathrm{dd}, J=10.8,7.0 \mathrm{~Hz}, 0.5 \mathrm{H}), 3.89$ (d, $J=6.4 \mathrm{~Hz}, 0.5 \mathrm{H}), 3.73(\mathrm{~s}, 1.5 \mathrm{H}), 3.71(\mathrm{~s}, 1.5 \mathrm{H}), 3.32 \sim$ $3.15(\mathrm{~m}, 4 \mathrm{H}), 0.92(\mathrm{~s}, 1.5 \mathrm{H}), 0.88(\mathrm{~s}, 1.5 \mathrm{H})$; ESI-HRMS calcd for $\mathrm{C}_{24} \mathrm{H}_{32} \mathrm{NO}_{3} 382.2377$, found 382.2370 .
6-苯甲酰基-3-四氢吡咯基-1,3,5(10)-三烯-17-酮-雌 甾(4m): $1: 2$ 混合物, 浅绿色固体; ${ }^{1} \mathrm{H}$ NMR $(400 \mathrm{MHz}$, $\left.\mathrm{CDCl}_{3}\right) \delta: 8.10 \sim 8.01(\mathrm{~m}, 0.66 \mathrm{H}), 7.90(\mathrm{~d}, J=7.3 \mathrm{~Hz}$, $1.33 \mathrm{H}), 7.62(\mathrm{t}, J=7.4 \mathrm{~Hz}, 0.33 \mathrm{H}), 7.53(\mathrm{dt}, J=7.3,2.4$ $\mathrm{Hz}, 1.33 \mathrm{H}), 7.43(\mathrm{t}, J=7.6 \mathrm{~Hz}, 1.33 \mathrm{H}), 7.25 \sim 7.19(\mathrm{~m}$, $1 \mathrm{H}), 6.49(\mathrm{td}, J=8.4,2.5 \mathrm{~Hz}, 1 \mathrm{H}), 6.13(\mathrm{~d}, J=2.2 \mathrm{~Hz}$, $0.66 \mathrm{H}), 6.08(\mathrm{~d}, J=2.4 \mathrm{~Hz}, 0.33 \mathrm{H}), 4.92(\mathrm{~d}, J=7.0 \mathrm{~Hz}$, $0.33 \mathrm{H}), 4.79$ (dd, $J=10.6,7.0 \mathrm{~Hz}, 0.66 \mathrm{H}), 3.20 \sim 3.05(\mathrm{~m}$, $4 \mathrm{H}), 0.91(\mathrm{~s}, 2.0 \mathrm{H}), 0.89(\mathrm{~s}, 1.0 \mathrm{H})$; ESI-HRMS calcd for $\mathrm{C}_{29} \mathrm{H}_{34} \mathrm{NO}_{2}$ 428. 2584, found 428. 2577.

6-萗甲酰基-3-四氢吡咯基-1,3,5(10)-三烯-17-酮-雌 甾(4n): $1: 1$ 混合物, 浅绿色固体; ${ }^{1} \mathrm{H}$ NMR $(400 \mathrm{MHz}$, $\left.\mathrm{CDCl}_{3}\right) \delta: 8.41 \sim 8.30(\mathrm{~m}, 0.5 \mathrm{H}), 8.23 \sim 8.14(\mathrm{~m}, 0.5 \mathrm{H})$, 7.97 (dd, $J=8.2,2.5 \mathrm{~Hz}, 1 \mathrm{H}), 7.93 \sim 7.85(\mathrm{~m}, 1 \mathrm{H}), 7.74(\mathrm{t}$, $J=7.0 \mathrm{~Hz}, 1 \mathrm{H}), 7.61 \sim 7.43(\mathrm{~m}, 3 \mathrm{H}), 7.25 \sim 7.19(\mathrm{~m}, 1 \mathrm{H})$, $6.53(\mathrm{dd}, J=8.6,2.5 \mathrm{~Hz}, 0.5 \mathrm{H}), 6.48(\mathrm{dd}, J=8.6,2.4 \mathrm{~Hz}$, $0.5 \mathrm{H}), 6.29 \sim 6.20(\mathrm{~m}, 1 \mathrm{H}), 4.89(\mathrm{dd}, J=10.6,7.2 \mathrm{~Hz}$, $0.5 \mathrm{H}), 4.75(\mathrm{~d}, J=6.9 \mathrm{~Hz}, 0.5 \mathrm{H}), 3.23 \sim 2.96(\mathrm{~m}, 4 \mathrm{H}), 0.88$ (s, $1.5 \mathrm{H}), 0.81$ (s, 1.5H); ESI-HRMS calcd for $\mathrm{C}_{33} \mathrm{H}_{36} \mathrm{NO}_{2}$ 478.2735, found 478.2741 .

辅助材料(Supporting Information) 化合物 3, 4a 4n 的核磁共振图谱. 这些材料可以免费从本刊网站 (http://sioc-journal.cn/)上下载.

\section{References}

[1] (a) Singh, S. M.; Gauthier, S.; Labrie, F. Curr. Med. Chem. 2000, 7 (2), 211.

(b) Labrie, F.; Belanger, A.; Cusan, L.; Labrie, C.; Simard, J.; LuuThe, V.; Diamond, P.; Gomez, J. L.; Candas, B. Endocr-Relat. Cancer 1996, 3, 243.

[2] (a) Njar, V. C. O. Synthesis 2000, 2019.

(b) Weidner, J. J.; Weintraub, P. M.; Schnettler, R. A.; Peet, N. P. Tetrahedron 1997, 53, 6303 .

(c) Coutts, I. G. C.; Southcott, M. R., J. Chem. Soc., Perkin. Trans. $11990,767$.

(d) Scherrer, R. A.; Beatty, H. R. J. Org. Chem. 1972, 37, 1681.

(e) Schon, U.; Messinger, J.; Buchholz, M.; Reinecker, U.; Thole, H.; Prabhu, M. K. S.; Konda, A. Tetrahedron Lett. 2005, 46, 7111.

(f) Zhang, X. Q.; Sui, Z. Tetrahedron Lett. 2003, 44, 3071.

(g) Radu, I. I.; Poirier, D.; Provencher, L. Tetrahedron Lett. 2002, 43, 7617.

[3] (a) Alvarez, F. S.; Ruiz, A. B. J. Org. Chem. 1965, 30, 2047. (b) Gold, A. M.; Schwenk, E. J. Am. Chem. Soc. 1959, 81, 2198.

[4] (a) Jing, Y.; Xu, C.-G.; Ding, K.; Lin, J.-R.; Jin, R.-H.; Tian, W.-S. Tetrahedron Lett. 2010, 51, 3242.

(b) Zheng, D.-Q.; Jing, Y.; Zheng, B.-Y.; Ye, Y.-F.; Xu, S.; Tian, W.-S.; Ma, H.-Y.; Ding, K. Tetrahedron 2016, 72, 2164.

(c) Yue, T.; Li, H.-P.; Ding, K. Tetrahedron Lett. 2016, 57, 4850.

[5] (a) Carey, F. A.; Sundberg, R. J. In Advanced Organic Chemistry, Part A: Structure and Mechanisms, 5th ed., Springer, New York, 2007, pp. $653 \sim 653$. 
(b) Carey, F. A.; Sundberg, R. J. In Advanced Organic Chemistry, Part B: Reactions and Synthesis, 5th ed., Springer, New York, 2007, pp. $46 \sim 55$.

[6] (a) Burn, D.; Cooley, G.; Davies, M. T.; Ducker, J. W.; Ellis, B.; Feather, P.; Hiscock, A. K.; Kirk, D. N.; Leftwick, A. P.; Petrow, V.;
Williamson, D. M. Tetrahedron 1964, 20, 597.

(b) Liisberg, S.; Godtfredsen, W. O.; Vangedal, S. Tetrahedron 1960, 9,149

(c) Fried, J. H.; Arth, G. E.; Sarett, L. H. J. Am. Chem. Soc. 1959 , 81,1235 .

(Zhao, C.) 